\title{
Ion Selectivity in P2X Receptors: a Comparison Between hP2X3 and zfP2X4
}

\author{
Vanesa Racigh, Gustavo Pierdominici-Sottile, and Juliana Palma* \\ Departamento de Ciencia y Tecnología, Universidad Nacional de Quilmes, Sáenz Peña 352, \\ Bernal B1876BXD, Argentina. Consejo Nacional de Investigaciones Científicas y Técnicas. \\ E-mail: juliana@unq.edu.ar \\ Phone: +54 (11) 43657100 ext 5657
}




\section{SUPPORTING INFORMATION}

\section{Methods}

\section{Models Construction and Equilibration}

\section{$\mathrm{P} 2 \mathrm{X} 4$}

The model of wild-type zfP2X4 was prepared as explained in Ref. 1. For completeness, we also provide the all the details here. The open form of zfP2X4, PDB entry 4DW1, ${ }^{2}$ has two main drawbacks. First, it lacks of the cytoplasmic part. Second, it presents intersubunit crevices at the TM domains that should not be present in membrane-embedded receptors. ${ }^{3}$ Previous MD simulations have demonstrated that lipid molecules can enter the channel through these crevices. ${ }^{3}$ To alleviate these limitations we built our models of open zfP2X 4 using the open form of hP2X3, PDB entry 5SVK, ${ }^{4}$ as a template for the TM and cytoplasmic regions. The next paragraphs describe how we assembled the model. The numbering of the residues and the names of the atoms used hereafter are those found in the 4DW1 and 5SV3 structures.

The sequences of zfP2X4 and hP2X3 were aligned with program Clustal Omega ${ }^{5}$ as provided by the Uniprot Web Page, http://www.uniprot.org/align/. To model the N-terminal region and most of the TM1 domain of zfP2X4, we superposed the $\mathrm{C}_{\alpha}$ atoms of L32, L33 and I34, belonging to TM1 of hP2X3, with the corresponding residues of zfP2X4: A40, L41 and V42. After that, the N-terminal part of zfP2X4 up to V42 was deleted and the backbone atoms of the corresponding $\mathrm{hP} 2 \mathrm{X} 3$ residues were added in their place. In this situation, the first residue of the $\mathrm{N}$ terminal region corresponds to F15 of zfP2X4 (F7 of hP2X3). Similarly, to model the C-terminal region and most of the TM2 domain of zfP2X4, the $\mathrm{C}_{\alpha}$ atoms of V326, A327 and A328, belonging to TM2 of hP2X3, were superposed with G342, A343 and G344, of zfP2X4. Then, the C-terminal region of zfP2X4 up to G342 was deleted and the backbone atoms of the corresponding hP2X3 residues were added in their place. This 
generates the conformation of TM2 up to D380 of zfP2X4 (E363 of hP2X3). After that, in those locations in which the two sequences differ, we mutated the residues of hP2X3 to recover the native sequence of zfP2X4. Besides, positions N78, N187 and H252 that had been modified to obtain the $4 \mathrm{DW} 1$ structure, ${ }^{2}$ were mutated back to the native sequence. We added residues D9 to C14 to the $\mathrm{N}$ terminal part and residues F381 to K389 to the C-terminal part, using program I-TASSER. ${ }^{6}$ The Leap module of the AMBER16 package ${ }^{7}$ concluded the preparation of the model by incorporating all the missing atoms of the edited PDB file. Therefore, while the crystal structure of open zfP2X4 consists in 324 residues, from R36 to I359, the model employed in the current calculations consisted of 381 residues, from D9 to K389. This model was soaked into a square lipid bilayer of $138.0 \AA^{2}$ with 256 POPC molecules in each layer. The graphical user interface of the CHARMM membrane builder ${ }^{8,9}$ was used for this purpose. The protein was displaced along the $\mathrm{Z}$ axis so that the top of the membrane was aligned with residue Y52.

The system was fed into the Leap module of AMBER16 where it was first neutralized. Water molecules were added to form an octahedral cell of $153.27 \times 150.477 \times 194.64 \AA$. Then $\mathrm{Na}^{+}$and $\mathrm{Cl}^{-}$ions were incorporated to produce a $0.15 \mathrm{M}$ concentration. The final model contains $248 \mathrm{Na}^{+}, 242 \mathrm{Cl}^{-}, 512$ POPC molecules and 89125 water molecules. Special care was devoted to introduce waters molecules into the extracellular vestibule and transmembrane channel. Otherwise either the simulations become unstable or the pore shrinks.

The Amber99SB force field ${ }^{10}$ was employed for the protein and water molecules while the Lipid14 force field was chosen for POPC. ${ }^{11}$

The SANDER and PMEMD modules of the AMBER16 package were employed to run the simulations. ${ }^{7}$ This initial structure was first minimized. Next, it was heated from $0 \mathrm{~K}$ to $100 \mathrm{~K}$ at NVT conditions during $120 \mathrm{ps}$. The aim of this stage is to achieve a temperature high enough to assure the proper functioning of the barostat. At that point, we changed from NVT to NPT conditions to allow the density to relax. We continued the heating for $1 \mathrm{~ns}$ until the system got to $303 \mathrm{~K}$. An harmonic restraint of $1.5 \mathrm{kcal} / \mathrm{mol} \AA^{2}$ was applied on the $\mathrm{C}_{\alpha}$ 
atoms of the protein and the oxygen atoms of the water molecules in the two heating periods. This was followed by four consecutive $5.0 \mathrm{~ns}$ simulations at $303 \mathrm{~K}$ in which the restraints were gradually diminished $\left(0.5,0.1,0.05\right.$ and $\left.0.01 \mathrm{kcal} / \mathrm{mol} \AA^{2}\right)$. A final equilibration stage of $2.5 \mathrm{~ns}$ was performed for the open structure while $7.0 \mathrm{~ns}$ were required for the closed structure. These times were needed to equilibrate the spacial distribution of the ions.

To build the mutants, we stripped the water molecules and ions from a PDB file corresponding to the wild-type model. The resulting structure was employed to create mutants D59N and D61N. Each of the variants was solvated again and ions were introduced to produce a neutral solution with a $0.15 \mathrm{M}$ concentration of $\mathrm{NaCl}$. These structures were then minimized, heated and equilibrated as explained above. The model of closed wild type zfP2X4 was prepared as detail in Ref. 12.

\section{P2X3}

The procedure we followed to build the computational models of open hP2X3 and its mutants is similar to those of zfP2X4. However, there are some details that just apply to this particular case. They are presented in the following paragraphs.

The initial structure was taken from the Protein Data Bank (PDB entry 5SVK). It lacks residues M1-D6 and T364-H397 at the N- and C-termini, respectively. We used the I-TASSER on-line server to incorporate them. ${ }^{6}$ The server afforded five alternative models. In four of them, the structure of the cytoplasmic cap was altered at the point where the missing segments were added. In the remaining case, the shape of the cytoplasmic cap was preserved. This was the structure employed for the construction of the models. The 5SVK structure has three mutations with regard to the wild-type receptor. These are T13P, S15V and V16I. Using the TLEAP module of AMBER16 we reversed the three mutations and incorporated all the missing atoms. ${ }^{7}$ The CHARMM-GUI interface ${ }^{9}$ was employed to soak the channel into a square lipid bilayer with 512 POPC molecules. The position of the channel with respect to the membrane was set according to the indications of Ref. 13. Using TLEAP, 
the system was placed in an octahedral cell of TIP3P water molecules. Finally, the required number of $\mathrm{Na}^{+}$and $\mathrm{Cl}^{-}$ions was added to produce a neutral system with a $0.15 \mathrm{M} \mathrm{NaCl}$ concentration. The dimensions of the final model are $136 \AA^{2}$ in the plane of the membrane and $165 \AA$ in the perpendicular direction. It contained $205 \mathrm{Na}^{+}$ions, $199 \mathrm{Cl}^{-}$and 73273 water molecules.

The system prepared as explained above was initially minimized. Then, it was heated from 0 to $80 \mathrm{~K}$ during $80 \mathrm{ps}$ using a Langevin thermostat with a collision frequency of $1.0 \mathrm{ps}^{-1}$. During this stage, volume was kept constant. Then, we switched from constant volume to constant pressure, allowing the density to relax for 0.9 ns. To this end, we employed a Berendsen barostat with a coupling constant of 2.0 ps. Once the density was equilibrated, we continued the heating until $\mathrm{T}=310 \mathrm{~K}$. As occurred with the simulations of the open zfP2X4 receptor, some restrictions were required to keep the model stable during the heating and equilibration stages. Harmonic restraints of $1.5 \mathrm{kcal} / \mathrm{mol} . \AA^{2}$ were applied on the $\mathrm{C}_{\alpha}$ of residues belonging to the TM2 segments, to avoid pore shrinking and its consequent dehydration. Besides, harmonic restraints of $1.0 \mathrm{kcal} / \mathrm{mol} \AA^{2}$ were applied to the center of mass of residues 154 to 157 of each chain to prevent the sinking of the channel into the membrane. These residues are located at the top of the extracellular domain, far away from the region of interest to the simulations $(>60 \AA)$. Water molecules, lipids and ions were allowed to move freely. After the heating was completed, the system was allowed to equilibrate for $15 \mathrm{~ns}$, gradually diminishing the restrains on TM2 $(1.5,1.0,0.5$, and 0.01 $\mathrm{kcal} / \mathrm{mol} \AA^{2}$ ). However, the restraints on the center of mass of residues 154 to 157 were maintained for the reasons explained below.

During one of the several preparation and trial runs in which the restraints had been removed, we observed that charged residues located at one of the left flippers got close to the membrane. When this happened, a Coulombic interaction developed between them and the lipids' heads. This interaction tilted the channel with respect to the vertical axis and moved it down, mostly at one side. This artifact was observed in a long simulation $(\sim 30 \mathrm{~ns})$ 
during which no other signs of instability were observed. To prevent this happening during the many production runs of native hP2X3 and its mutants, we decided to keep the restraints on residues 154 to 157 . With this setting, we never observed the aforementioned behavior in any of the 40 standard MD simulations used for production, which accumulate 2,5 $\mu s$ of simulation time, nor in the more than 140 shorter US simulations, which accumulate 1,6 $\mu s$. We built the mutants of hP2X3 using a protocol similar to that of zfP2X4. In this case, we prepared three mutants: E46Q, D53N, and E57Q. The model of closed wild type hP2X3 was built, heated and equilibrated following similar guidelines. The initial structure was taken from the Protein Data Bank, PDB entry 5SVJ. Note that this structure lacks the cytoplasmic cap and we did not add any residue to complete it. For this model, the size of the octahedral box is $133 \times 136 \times 145 \AA$.It contains $166 \mathrm{Na}^{+}, 175 \mathrm{Cl}^{-}, 512 \mathrm{POPC}$ molecules and 61029 water molecules.

The interactions in all the systems were modeled with the Amber99SB force field ${ }^{10}$ in the case of the protein, ions and water, plus the Lipid14 force field ${ }^{11}$ for the POPC molecules. The simulations were run with the PMEMD module of the AMBER16 package. Bonds involving hydrogen atoms were constrained with the SHAKE algorithm. ${ }^{14}$ Accordingly, the time step could be set to 2.0 fs. A cutoff radius of $10.0 \AA$ was imposed to the non-bonded interactions, and the Particle Mesh Ewald method ${ }^{15,16}$ was employed to calculate the Coulombic interactions. According to the default procedure of CHARMM-GUI, the axis perpendicular to the membrane was set as the z-axis of the model. The origin of the coordinate system was set as the mass center of the $\mathrm{C}_{\alpha}$ atoms of residues 45 to 54 . This point is located at the bottom of the extracellular vestibule and above the transmembrane helix (see Fig. 1).

\section{Standard MD simulations}

For each computational model, except that of wild-type hP2X3, we run 10 independent simulations of 50 ns. All these simulations were started from the last snapshot of the equilibration stage. For wild-type hP2X3, a more extensive sampling was required since these simulations 
were also used to estimate parts the PMF for the passage of $\mathrm{Na}^{+}$and $\mathrm{Cl}^{-}$across the pore. Accordingly each of the 10 simulations lasted for $100 \mathrm{~ns}$, giving a total sampling of $1.0 \mu \mathrm{s}$. In all cases, the initial velocities were randomly chosen from a Maxwellian distribution. Samples were collected every 0.1 ns. Trajectories were visualized using VMD. ${ }^{17}$

\section{Umbrella Sampling Simulations}

Umbrella sampling simulations were run for the computational model of wild-type hP2X3 to determine the PMF for the passage of $\mathrm{Na}^{+}$and $\mathrm{Cl}^{-}$along the pore. We observed in standard MD simulations that $\mathrm{Na}^{+}$ions enter the receptor from both the extracellular and intracellular domains and partially penetrate the pore. However, they only occasionally attain the narrowest part. Thus, there is a fair chance to find $\mathrm{Na}^{+}$inside the channel for $Z \leq 14 \AA$ and $Z \geq 26 \AA$, but the probabilities between these limits are rather low. Accordingly, for $\mathrm{Na}^{+}$, the US algorithm is strictly needed for $14 \AA \leq Z \leq 26 \AA$. Nevertheless, we run US simulations for a larger range in order to superimpose the probability profiles calculated from US with those of standard simulations in the regions where they overlap. Thus, US simulations for $\mathrm{Na}^{+}$were run for $0 \AA \leq Z \leq 30 \AA$. This combined strategy was crucial to get the PMF for $Z \geq 30 \AA$ where the US simulations are extremely hard to converge. Applying the same criterion, the US simulations for $\mathrm{Cl}^{-}$were run for $0 \leq \AA$ $Z \leq 40 \AA$. In this case, we have to get to larger values of $Z$ because $\mathrm{Cl}^{-}$ions are much less prone to enter the channel from the cytoplasmic side.

In both cases, the centers of the US windows were separated by $1.0 \AA$. The force constant of the harmonic bias potential was set to $4.0 \mathrm{kcal} / \mathrm{mol} \AA^{2}$. For the US windows located in the extracellular vestibule, we applied a cylindrical restriction with $r=14.5 \AA$ to the ion subjected to the bias potential. In US simulations of $\mathrm{Cl}^{-}$, we also applied a cylindrical

restriction for $35 \AA \leq Z \leq 40 \AA$. In this case, the radius was set to $25.0 \AA$. Finally, when the ion subjected to the bias potential was within the pore, a distance restriction with $d=10 \AA$ was applied to the remaining ions, so that it traverses the pore under single-ion conditions. ${ }^{18}$ 
We run 8 independent US simulations for windows with $0 \AA \leq Z \leq 34 \AA$ and 16 for $35 \AA \leq$ $Z \leq 40 \AA$. Note that the later only applies to $\mathrm{Cl}^{-}$. In each case, the final structure of the simulation at a given window was used as the initial structure for the next one. We sampled 2 ns per window but the first 0.2 ns were considered as an equilibration period and were therefore discarded. Accordingly, the total sampling amounts to $14.4 \mathrm{~ns}$ for windows at 0 $\AA \leq Z \leq 34 \AA$ and 28.8 ns for $35 \AA \leq Z \leq 40 \AA$. 


\section{SI Figures}

\begin{tabular}{|c|c|}
\hline MOUSE & MAAAQPRLPAGAAMVRRLARGCWSAFWDYETPKVIVVRNRRLGFVHRMVQLLILLYFVWY 60 \\
\hline DANRE & MAPRVLGFIKGFFVYETAKSVVVKSWSVGIINRIVQLLIILYFICW 46 \\
\hline BOVIN & ......................... MNCISDFFTYETTKSVVVKSWTIGIINRAVQLLIISYFVGW 41 \\
\hline CAPHI & .......................... 41 \\
\hline RAT & 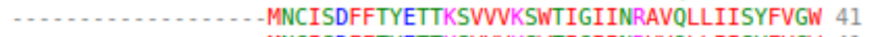 \\
\hline HUMAN & . . . MNCISDFFTYETTKSVVVKSWTIGIINRVVQLLIISYFVGW 41 \\
\hline LOXAF & . . . MNCISDFFTYETTKSVVVKSWTIGIINRAVQLLIISYFVGW 41 \\
\hline PIG & ......................... MNCISDFFTYETTKSVVVKSWTIGIINRAVQLLIISYFVGW 41 \\
\hline CANLF & 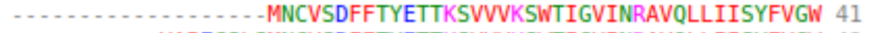 \\
\hline AILME & 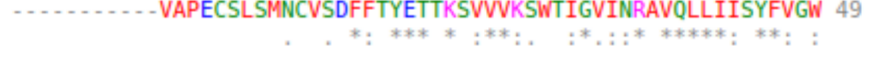 \\
\hline MOUSE & VFIVQKSYQDSETGPEFSIITKVKGITMSEHKVWDVEEYVKPPEGGSVVSIITRIEVTPS 120 \\
\hline DANRE & VFMHEKAHQLRDTGIESAVMTKVKGLGNFNDRVMDVADYVIPSQGASSFSIITNMVVTAN 106 \\
\hline BOVIN & VFLHEKAYQVRDTAIESSVVTKVKGFGRYANRVMDVSDYVTPPQGTSVFVIITKMIVTEN 101 \\
\hline CAPHI & VFLHEKAYQVRDTAIESSVVTKVKGFGRYANRVMDVSDYVTPPQGTSVFVIITKMIVTEN 101 \\
\hline RAT & VFLHEKAYQVRDTAIESSVVTKVKGFGRYANRVMDVSDYVTPPQGTSVFVIITKMIVTEN 101 \\
\hline HUMAN & VFLHEKAYQVRDTAIESSVVTKVKGSGLYANRVMDVSDYVTPPQGTSVFVIITKMIVTEN 101 \\
\hline LOXAF & VFLHEKAYQVRDTAIESSVVTKVKGFGRYANRVMDVSDYVTPPQGTSVFVIITKMIATEN 101 \\
\hline PIG & VFLHEKAYQVRDTAIESSVVTKVKGFGRYANRVMDVSDYVTPPQGTSVFVIITKMIVTEN 101 \\
\hline CANLF & VFLHEKAYQVRDTAIESSVVTKVKGFGRYANRVMDVSDYVTPPQGTSVFVIITKMIVTEN 101 \\
\hline \multirow[t]{2}{*}{ AILME } & VFLHEKAYQVRDTAIESSVVTKVKGFGRYANRVMDVSDYVTPPQGTSVFVIITKMIVTEN 109 \\
\hline & $\begin{array}{r}{ }^{* *}::^{*}::^{*}:^{*},{ }^{* *}:::^{* * * * *} \\
\text { (a) }{ }^{* * * *}::^{* *}:^{* * *},{ }^{* * *},: *^{*},\end{array}$ \\
\hline DANRE & - -MSESVGCCDSVSQCFFDYYTSKILIIRSKKVGTLNRFTQALVIAYVIGYVCVYNKGYQ 58 \\
\hline LOXAF & - . - - LAGCCAVLAGFLFEYDTPRIVLIRSRKVGLMNRAVQLLILAYVIGWVFVWEKGYQ 55 \\
\hline RAT & - . - MAGCCSVLGSFLFEYDTPRIVLIRSRKVGLMNRAVQLLILAYVIGWVFVWEKGYQ 55 \\
\hline MOUSE & - . - - MAGCCSVLRAFLFEYDTPRIVLIRSRKVGLMNRVVQLLILAYVIGWVFVWEKGYQ 55 \\
\hline HUMAN & - . - MAGCCAALAAFLFEYDTPRIVLIRSRKVGLMNRAVQLLILAYVIGWVFVWEKGYQ 55 \\
\hline CANLF & - - - - MAGCCAALAPFLFEYDTPRIVLIRSRKVGLMNRAVQLLILAYVIGWVFVWEKGYQ 55 \\
\hline AILME & PSPGPRPEAQHRLLAFLFEYDTPRIVLIRSRKVGLMNRAVQLLILAYVIGWVFVWEKGYQ 60 \\
\hline PIG & - - - MAGCCAVLYAFLFEYDTPRIVLIRSRKVGLMNRMVQLLILAYVIGWVFVWEKGYQ 55 \\
\hline BOVIN & - - - - MTGCCTVLGAFLFEYDTPRIVLIRSRKVGLMNRTVQLLILAYVIGWVFVWEKGYQ 55 \\
\hline \multirow[t]{2}{*}{ CAPHI } & - MAGCCAVLGAFLFEYDTPRIVLIRSRKVGLMNRTVQLLILAYVIGWVFVWEKGYQ 55 \\
\hline & . $\quad: \quad:^{*}: * *:^{*}::^{* * *}: * * * \quad:^{* *} \cdot{ }^{*} *::^{* * * * *}:^{*} *::^{* * * *}$ \\
\hline DANRE & DDTVLSSVTTKVKGIALTNTSELGERIWDVADYII - PPQEDGSFFVLTNMIITTNQTQS 117 \\
\hline LOXAF & E DSVVSSVTIKAKGVAVTNTSELGFRIWDVADYVMLQAQEENSIFIMTNMILTMNQTQG 115 \\
\hline RAT & ENDSVVSSVTTKAKGVAVTNTSQLGFRIWDVADYVI - PAQEENSLFIMTMMIVTVNQTQS 114 \\
\hline MOUSE & ENDSVVSSVTTKAKGVAVTNTSQLGFRIWDVADYVV - PAQEENSLFIMTMMIVTVNQTQG 114 \\
\hline HUMAN & ENDSVVSSVTTKVKGVAVTNTSKLGFRIWDVADYVI - PAQEENSLFVMTNVILTMNQTQG 114 \\
\hline CANLF & ENDSVVSSVTTKAKGVTVTNTSILGFRVWDVADYVV - PVQEENSLFIMTNMIITLNQTQG 114 \\
\hline AILME & ENDSVVSSVTTKAKGVTVTNTSTLGFRVWDVADYVI - PAQEENSLFVMTNMIITMNQTQG 119 \\
\hline PIG & ENDSVVSSVTTKAKGVTMTNTSKLGFRIWDVADYVI - PAQEENSLFIMTNLIITMNQTQG 114 \\
\hline BOVIN & ENDSVVSSVTVKAKGVTMTNTSKLGFRIWDVADYVI - PAQEENSVFIMTNMVITMNQTQG 114 \\
\hline \multirow[t]{2}{*}{ CAPHI } & EfDSVVSSVTVKAKGVTMTNTSKLGFRIWDVADYVI - PAQEENSVFIMTNMVITMNQTQG 114 \\
\hline & $: * *: *: * * * * * * ; *::: * * * * * * * ;: * * * * * *:: \quad * *: *^{*}, *:: * *::: * * * * * *$ \\
\hline
\end{tabular}

Figure S1: Alignments of several members of the P2X3 and P2X4 subfamilies. Residues whose role was studied in this work are put within a black rectangle. (a) P2X3, (b) P2X4. 


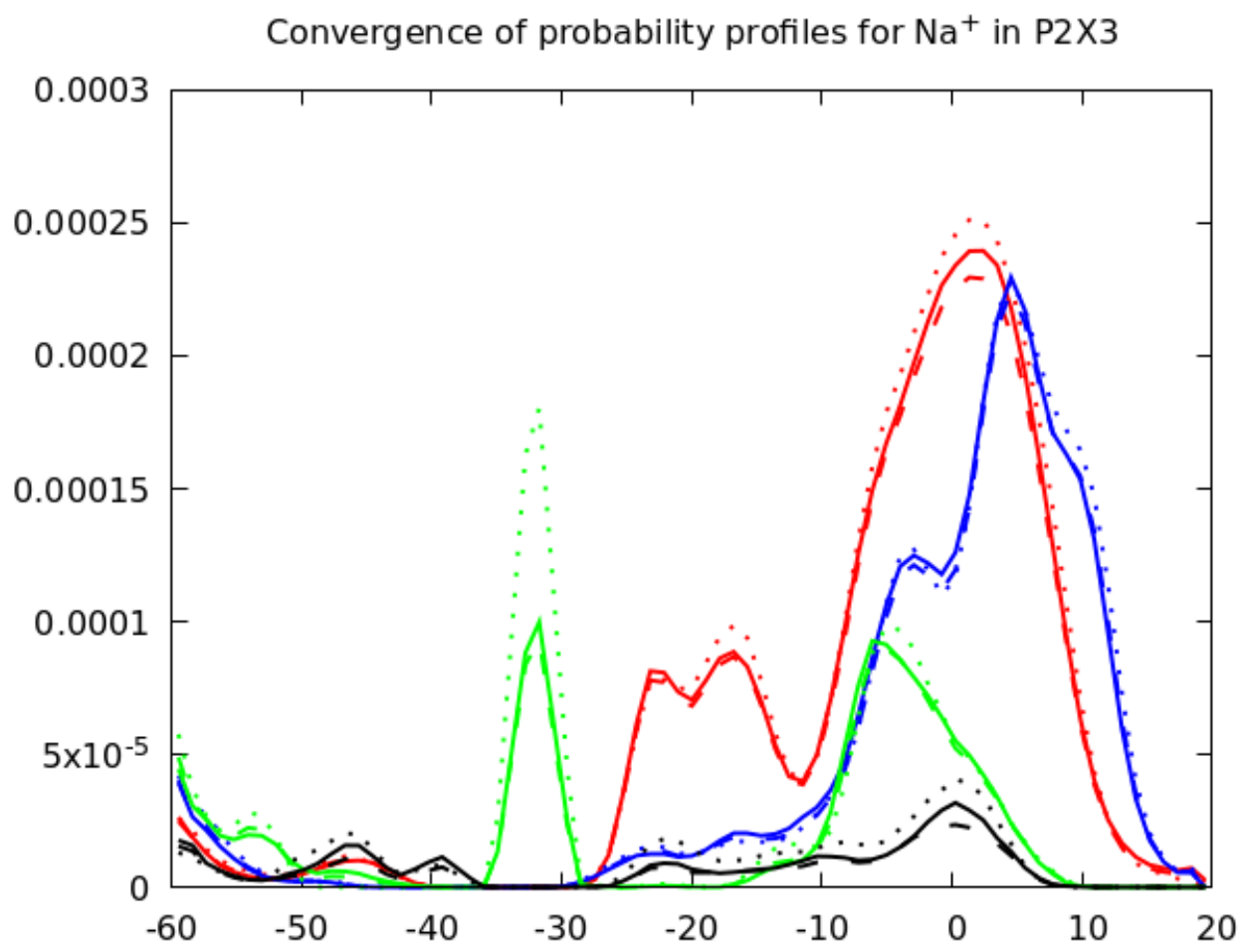

Figure S2: Convergence of the probability profiles for $\mathrm{Na}^{+}$in $\mathrm{hP} 2 \mathrm{X} 3$ and its mutants. Dotted lines are employed for probabilities computed with half of the simulation time. Dashed lines are employed for probabilities computed with $3 / 4$ of the whole simulation time. Solid lines correspond to the results obtained with all the data. Different colors correspond to alternative models according to the following scheme: $\mathrm{WT}=$ red, E46Q $=$ blue, E57Q green, D53N black. 


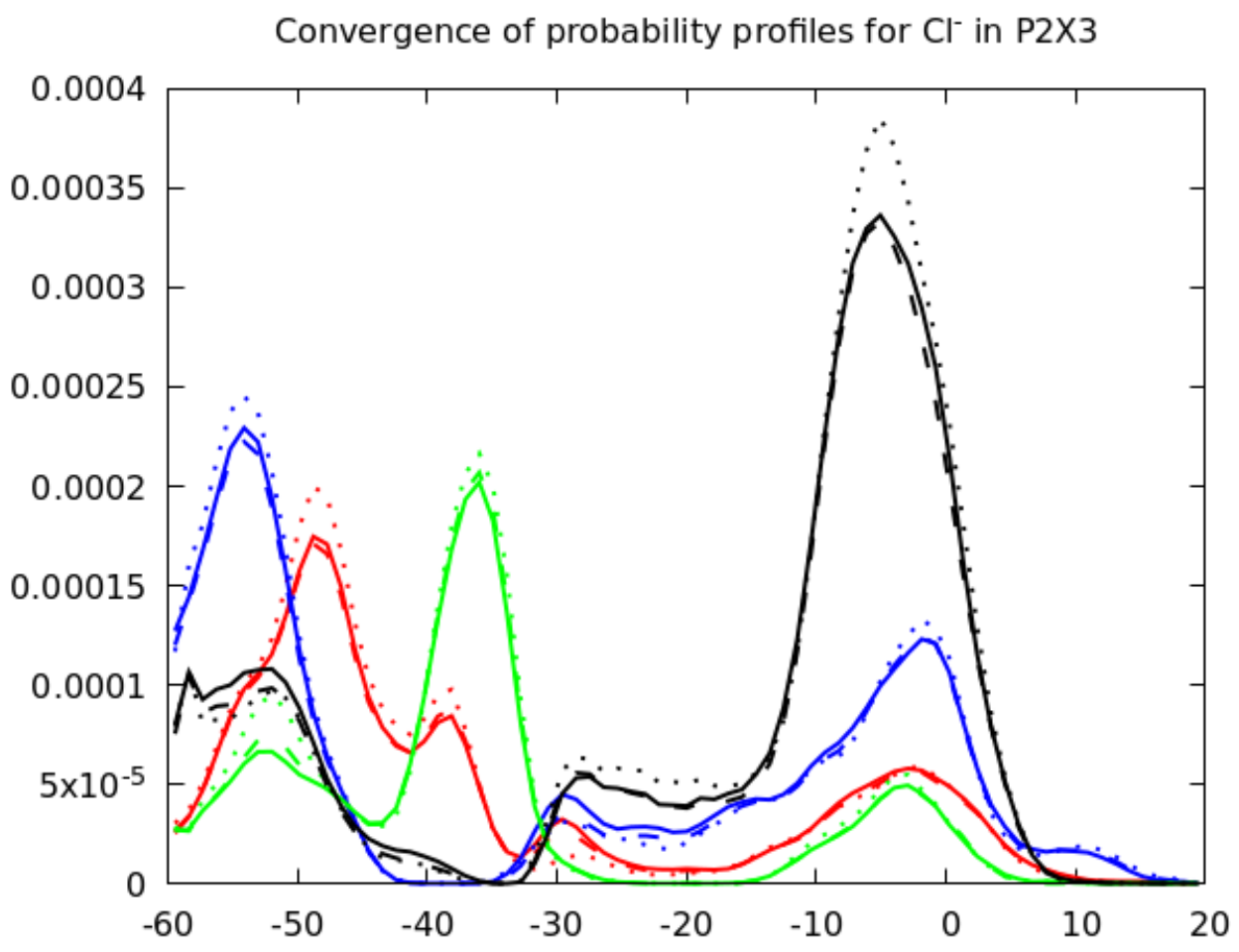

Figure S3: Convergence of the probability profiles for $\mathrm{Cl}^{-}$in hP2X3 and its mutants. Dotted lines are employed for probabilities computed with half of the simulation time. Dashed lines are employed for probabilities computed with $3 / 4$ of the whole simulation time. Solid lines correspond to the results obtained with all the data. Different colors correspond to alternative models according to the following scheme: $\mathrm{WT}=$ red, E46Q $=$ blue, E57Q green, D53N black. 


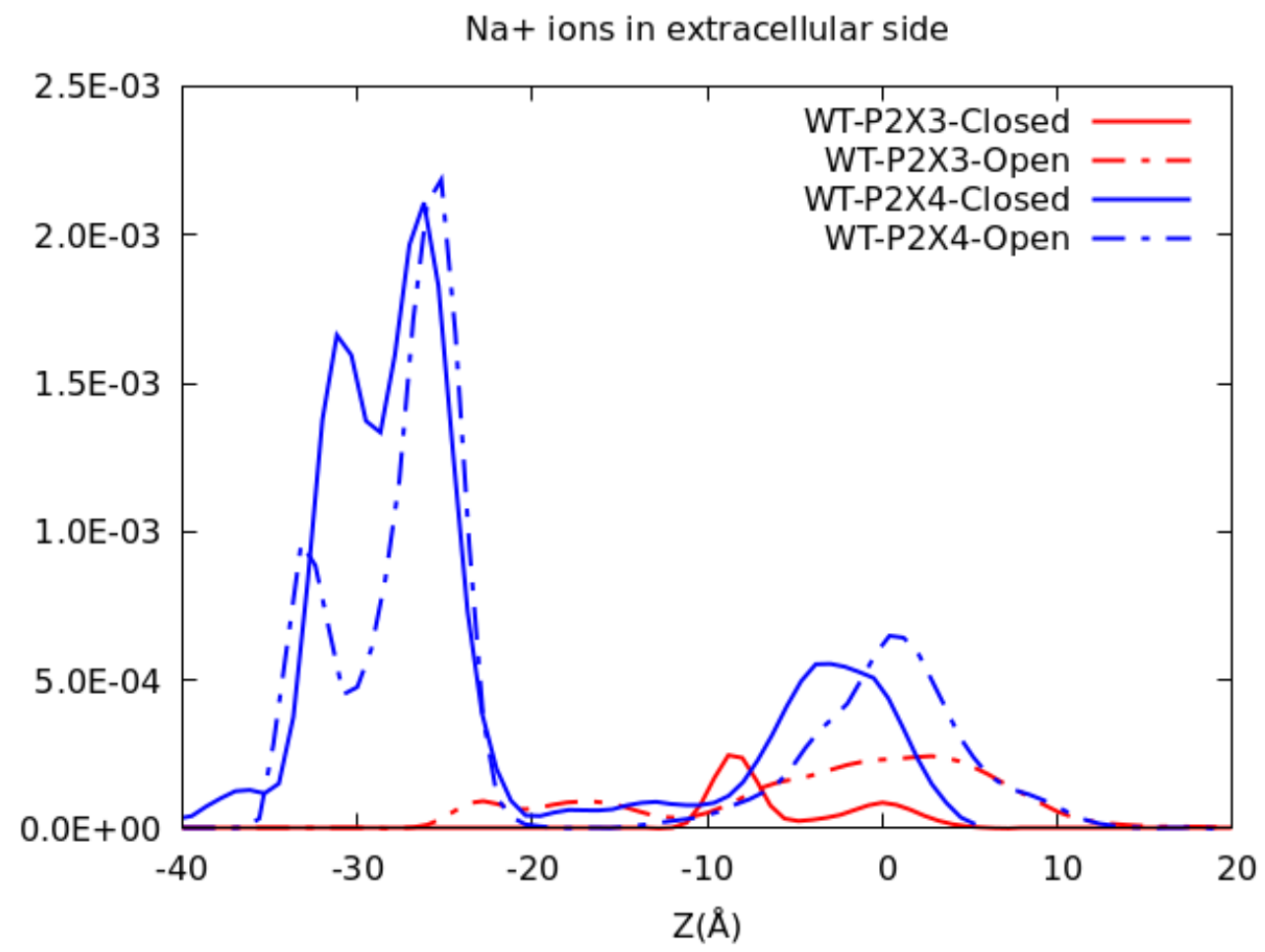

Figure S4: Comparison of the probability densities for $\mathrm{Na}^{+}$in wild type zfP2X4 and hP2X3. Dashed lines are employed for the open channels, solid lines are employed for the closed ones. 


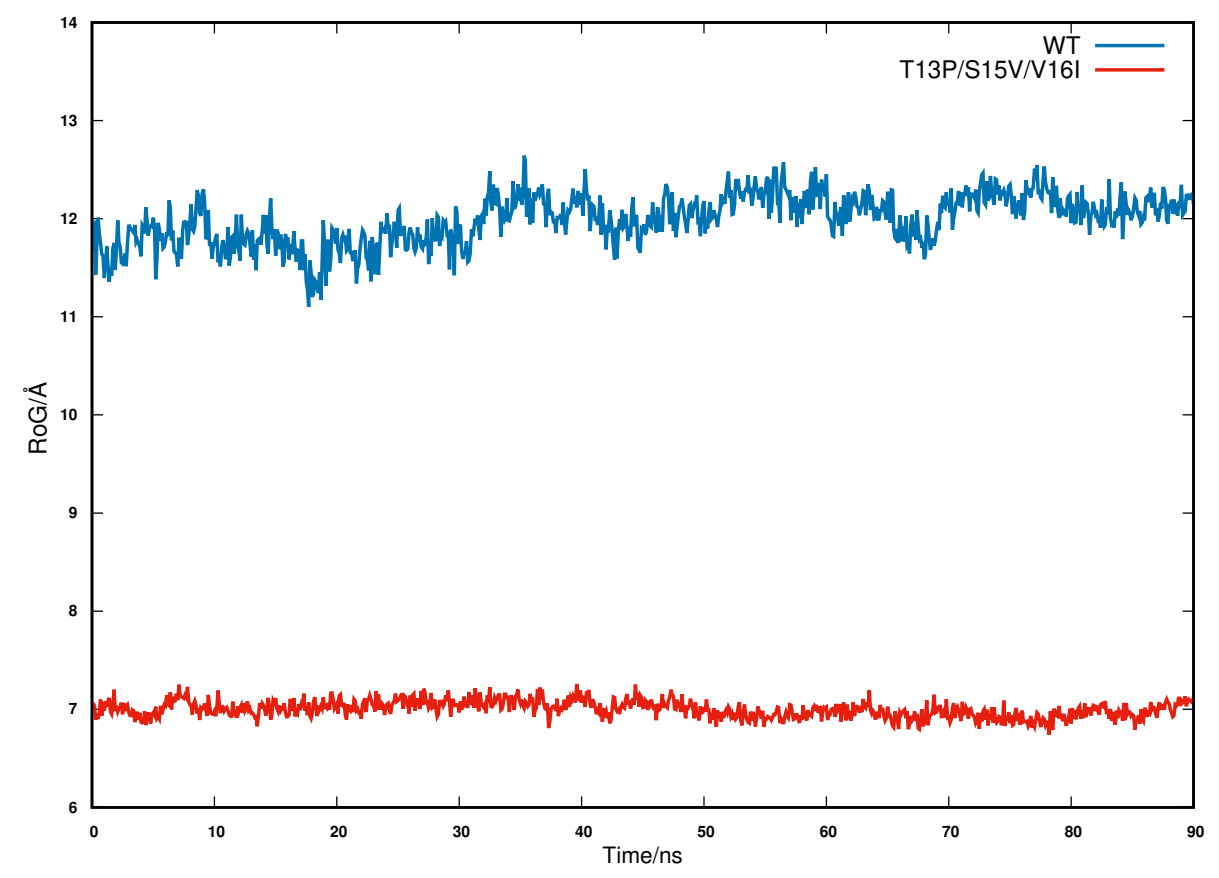

Figure S5: Radius of gyration for the core of residues that stabilize the cytoplasmic cap. The results for two models are compared. The curve in red corresponds to a model directly obtained from the PDB structure of open hP2X3. It contains the mutations T13P, S15V, V16I. The curve in blue was obtained with the wild type structure, obtained by reversing the mutations introduced to capture the open state. 


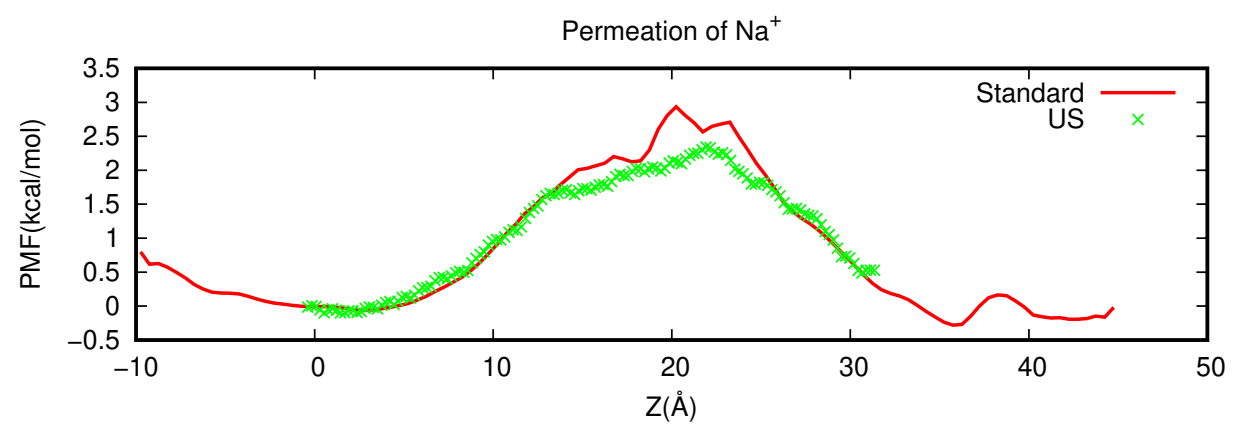

Permeation of $\mathrm{Cl}^{-}$

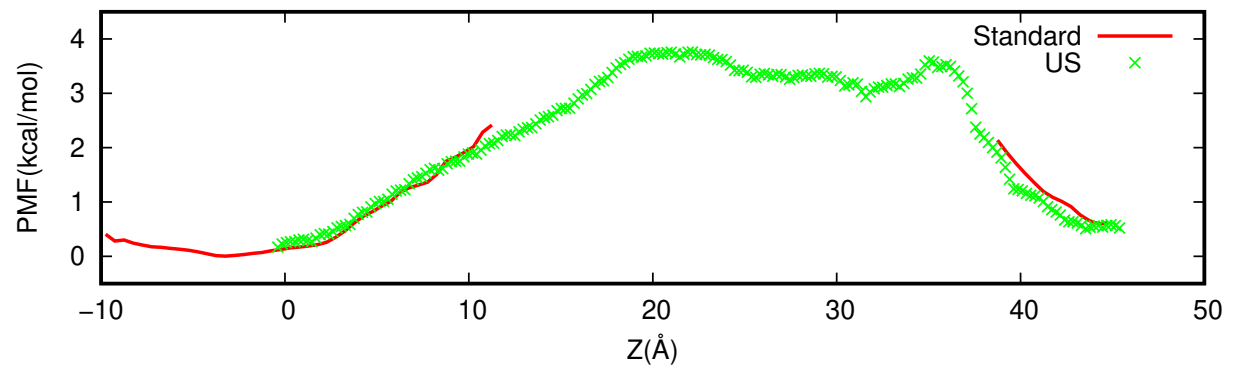

Figure S6: Olverlap between the PMFs computed from US and unbiased (standard) MD simulations. 

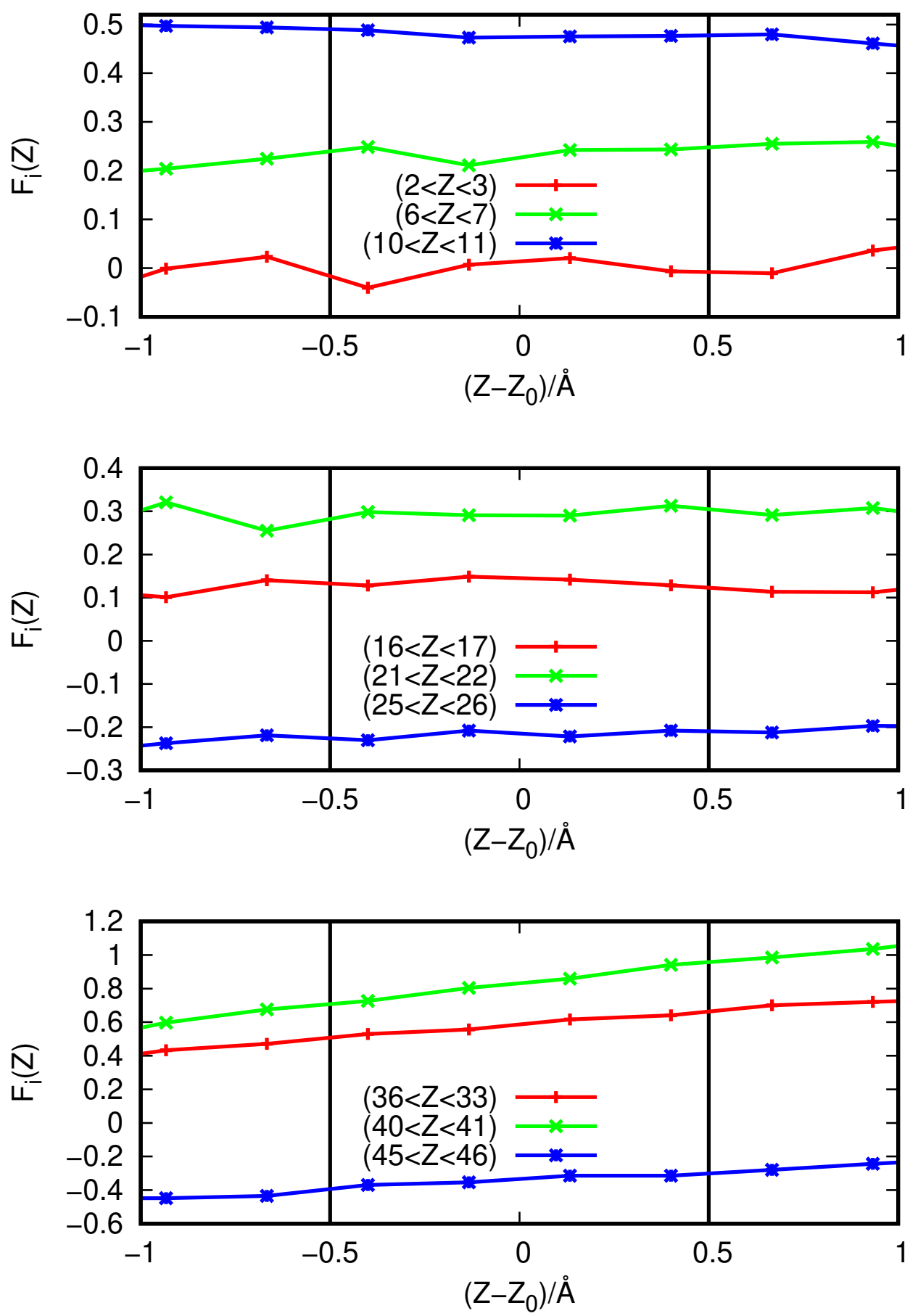

Figure S7: Convergence of the US simulations for $\mathrm{Na}^{+}$at different locations along the $Z$-axis. In order to have several curves on the same chart, $F_{i}(Z)$ was plotted as a function of $Z-Z_{0}$, where $Z_{0}$ denotes the center of the interval under consideration. Function $F_{i}(Z)$ should not show a drift in the range $-0.5-0.5$. 

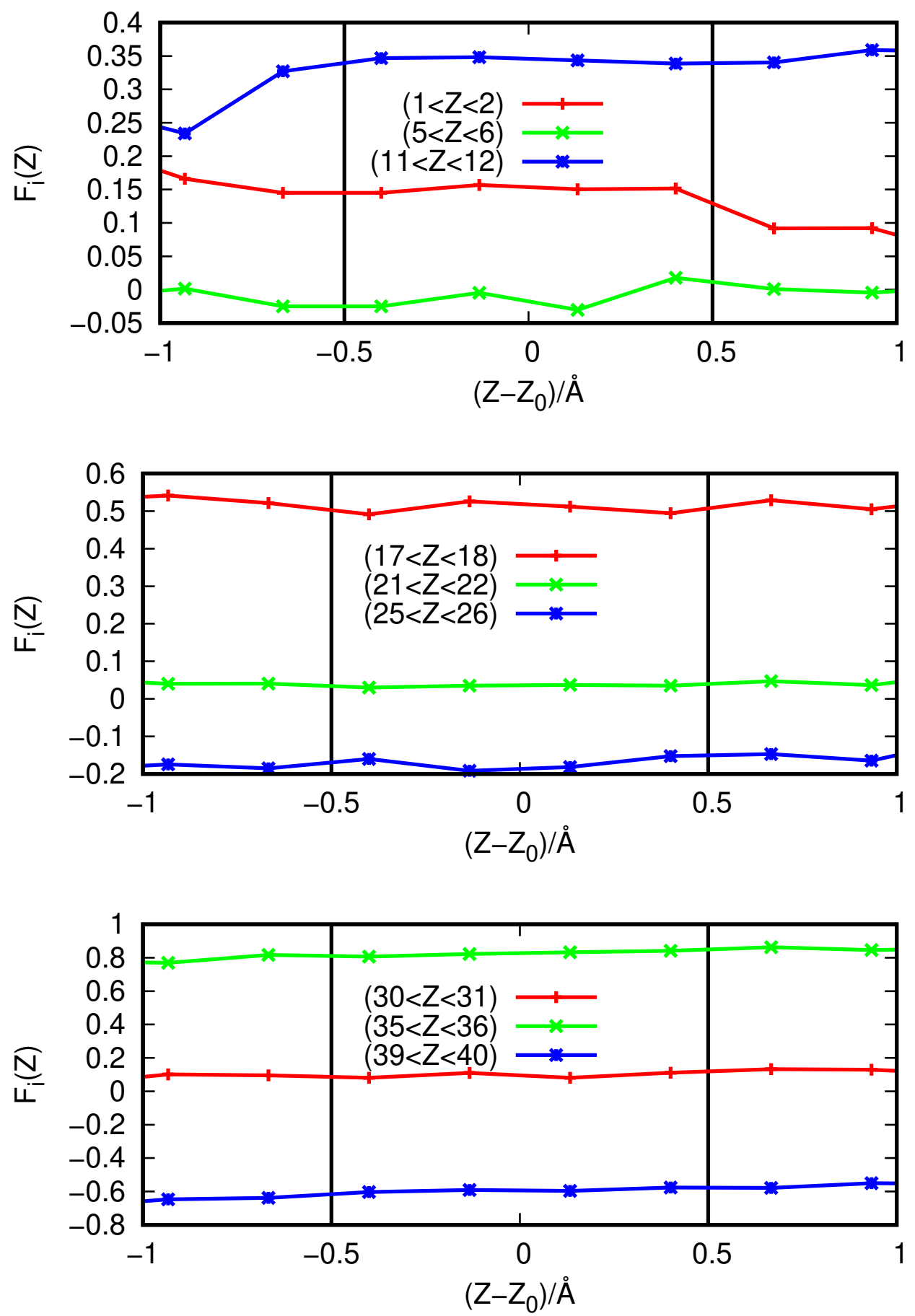

Figure S8: Convergence of the US simulations for $\mathrm{Cl}^{-}$at different locations along the $Z$-axis. In order to have several curves on the same chart, $F_{i}(Z)$ was plotted as a function of $Z-Z_{0}$, where $Z_{0}$ denotes the center of the interval under consideration. Function $F_{i}(Z)$ should not show a drift in the range $-0.5-0.5$. 


\section{SI Movies}

- Movie S1 shows part of a simulation of wild type P2X3 in which a $\mathrm{Na}^{+}$ion travels along the pore of the channel.

\section{References}

(1) Pierdominici-Sottile, G.; Racigh, V.; Ormazábal, A.; Palma, J. Charge Discrimination in P2X4 Receptors Occurs in Two Consecutive Stages. The Journal of Physical Chemistry B 2019, 123, 1017-1025.

(2) Hattori, M.; Gouaux, E. Molecular Mechanism of ATP Binding and Ion Channel Activation in P2X Receptors. Nature 2012, 485, 207-212.

(3) Heymann, G.; Dai, J.; Li, M.; Silberberg, S. D.; Zhou, H.-X.; Swartz, K. J. Inter and Intrasubunit Interactions Between Transmembrane Helices in the Open State of P2X Receptor Channels. Proceedings of the National Academy of Sciences 2013, 110, 4045-4054.

(4) Wang, J.; Wang, Y.; Cui, W.-W.; Huang, Y.; Yang, Y.; Liu, Y.; Zhao, W.-S.; Cheng, X.Y.; Sun, W.-S.; Cao, P. et al. Druggable negative allosteric site of P2X3 receptors. Proceedings of the National Academy of Sciences 2018, 115, 4939-4944.

(5) Sievers, F.; Wilm, A.; Dineen, D.; Gibson, T. J.; Karplus, K.; Li, W.; Lopez, R.; McWilliam, H.; Remmert, M.; Söding, J. et al. Fast, Scalable Generation of HighQuality Protein Multiple Sequence Alignments Using Clustal Omega. Molecular Systems Biology 2011, 7 .

(6) Roy, A.; Kucukural, A.; Zhang, Y. I-TASSER: a Unified Platform for Automated Protein Structure and Function Prediction. Nature Protocols 2010, 5, 725-738. 
(7) Case, D.; Darden, T.; Cheatham, T.; Simmerling, C.; Wang, J.; Duke, R.; Luo, R.; Walker, R.; Zhang, W.; Merz, K. et al. Amber16, Reference Manual. 2016.

(8) Jo, S.; Lim, J.; Klauda, J. B.; Im, W. CHARMM-GUI Membrane Builder for Mixed Bilayers and its Application to Yeast Membranes. Biophysical journal 2009, 97, 50-58.

(9) Wu, E. L.; Cheng, X.; Jo, S.; Rui, H.; Song, K. C.; Dávila-Contreras, E. M.; Qi, Y.; Lee, J.; Monje-Galvan, V.; Venable, R. M. et al. CHARMM-GUI Membrane Builder Toward Realistic Biological Membrane Simulations. Journal of Computational Chemistry 2014, 35, 1997-2004.

(10) Hornak, V.; Abel, R.; Okur, A.; Strockbine, B.; Roitberg, A.; Simmerling, C. Comparison of Multiple Amber Force Fields and Development of Improved Protein Backbone Parameters. Proteins: Structure, Function, and Bioinformatics 2006, 65, 712-725.

(11) Dickson, C. J.; Madej, B. D.; Skjevik, A. A.; Betz, R. M.; Teigen, K.; Gould, I. R.; Walker, R. C. Lipid14: The Amber Lipid Force Field. Journal of Chemical Theory and Computation 2014, 10, 865-879.

(12) Pierdominici-Sottile, G.; Moffatt, L.; Palma, J. The Dynamic Behavior of the P2X4 Ion Channel in the Closed Conformation. Biophysical Journal 2016, 111, 2642-2650.

(13) Mansoor, S. E.; Lü, W.; Oosterheert, W.; Shekhar, M.; Tajkhorshid, E.; Gouaux, E. X-ray Structures Define Human P2X3 Receptor Gating Cycle and Antagonist Action. Nature 538(7623), 66-71.

(14) Miyamoto, S.; Kollman, P. A. Settle: An analytical version of the SHAKE and RATTLE algorithm for rigid water models. Journal of Computational Chemistry 1992, 13, 952962.

(15) Darden, T.; York, D.; Pedersen, L. Particle Mesh Ewald: An N.log(N) Method for 
Ewald Sums in Large Systems. The Journal of Chemical Physics 1993, 98, 1008910092.

(16) Essmann, U.; Perera, L.; Berkowitz, M. L.; Darden, T.; Lee, H.; Pedersen, L. G. A Smooth Particle Mesh Ewald Method. The Journal of Chemical Physics 1995, 103, $8577-8593$.

(17) Humphrey, W.; Dalke, A.; Schulten, K. VMD - Visual Molecular Dynamics. Journal of Molecular Graphics 1996, 14, 33-38.

(18) Roux, B. Statistical Mechanical Equilibrium Theory of Selective Ion Channels. Biophysical Journal 1999, 77, $139-153$. 